\title{
The role of lysosomes in cancer development and progression
}

\author{
Tao Tang ${ }^{1 \dagger}$, Ze-yu Yang ${ }^{1 \dagger}$, Di Wang ${ }^{1}$, Xian-yan Yang ${ }^{1}$, Jun Wang ${ }^{1}$, Lin Li ${ }^{1}$, Qian Wen ${ }^{1}$, Lei Gao ${ }^{2}$, Xiu-wu Bian \\ and Shi-cang $\mathrm{Yu}^{1 *}$ (i)
}

\begin{abstract}
Lysosomes are an important component of the inner membrane system and participate in numerous cell biological processes, such as macromolecular degradation, antigen presentation, intracellular pathogen destruction, plasma membrane repair, exosome release, cell adhesion/migration and apoptosis. Thus, lysosomes play important roles in cellular activity. In addition, previous studies have shown that lysosomes may play important roles in cancer development and progression through the abovementioned biological processes and that the functional status and spatial distribution of lysosomes are closely related to cancer cell proliferation, energy metabolism, invasion and metastasis, immune escape and tumor-associated angiogenesis. Therefore, identifying the factors and mechanisms that regulate the functional status and spatial distribution of lysosomes and elucidating the relationship between lysosomes and the development and progression of cancer can provide important information for cancer diagnosis and prognosis prediction and may yield new therapeutic targets. This study briefly reviews the above information and explores the potential value of lysosomes in cancer therapy.
\end{abstract}

Keywords: Lysosomes, Cancer, Metastasis, Energy metabolism, Spatial distribution

\section{Background}

\section{Introduction to the lysosome}

Lysosomes are an important component of the inner membrane system. This organelle was first discovered by Christian de Duve in 1955 and was so named because it contains a variety of hydrolases. Precursors of lysosomal enzymes are synthesized in the rough endoplasmic reticulum $(\mathrm{rER})$ and then migrate to the cis-Golgi, where mannose residues on the oligosaccharide chain are phosphorylated to form mannose-6-phosphate (M-6-P), an important sorting signal for lysosomal enzymes. In the trans-Golgi network (TGN), phosphorylated lysosomal

\footnotetext{
*Correspondence: yushicang@163.com

${ }^{\dagger}$ Tao Tang and Ze-yu Yang have contributed equally to the study.

1 Department of Stem Cell and Regenerative Medicine, Institute of Pathology and Southwest Cancer Center, Southwest Hospital, Third Military Medical University (Army Medical University), Chongqing 400038, China

Full list of author information is available at the end of the article
}

enzymes bind to M-6-P receptors, which direct the enzymes into clathrin-coated vesicles. Then, the clathrin lattice is depolymerized into subunits. The uncoated transport vesicles can fuse with autophagosome or heterophagosome to form autophagolysosome, heterophagic lysosome or phagolysosome. Lysosomes were previously believed to be the sites of the degradation of intracellular and extracellular substances. Therefore, researchers called lysosomes the "garbage disposals" of cells [1]. However, more in-depth studies showed this viewpoint to be too one-sided. Emerging evidence suggests that lysosomes may also be the cellular center for intracellular transport (Fig. 1), signaling (Fig. 2), and metabolism.

\section{Lysosomes play a crucial role in intracellular transport}

The endosome-lysosome pathway is the primary means by which materials are trafficked and exchanged within the cell. Exogenous materials can be delivered to lysosomes by endocytosis and phagocytosis. Vesicles

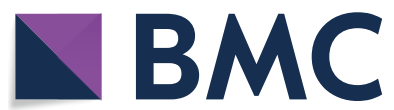

(c) The Author(s) 2020. This article is licensed under a Creative Commons Attribution 4.0 International License, which permits use, sharing, adaptation, distribution and reproduction in any medium or format, as long as you give appropriate credit to the original author(s) and the source, provide a link to the Creative Commons licence, and indicate if changes were made. The images or other third party material in this article are included in the article's Creative Commons licence, unless indicated otherwise in a credit line to the material. If material is not included in the article's Creative Commons licence and your intended use is not permitted by statutory regulation or exceeds the permitted use, you will need to obtain permission directly from the copyright holder. To view a copy of this licence, visit http://creativeco mmons.org/licenses/by/4.0/. The Creative Commons Public Domain Dedication waiver (http://creativecommons.org/publicdomain/ zero/1.0/) applies to the data made available in this article, unless otherwise stated in a credit line to the data. 


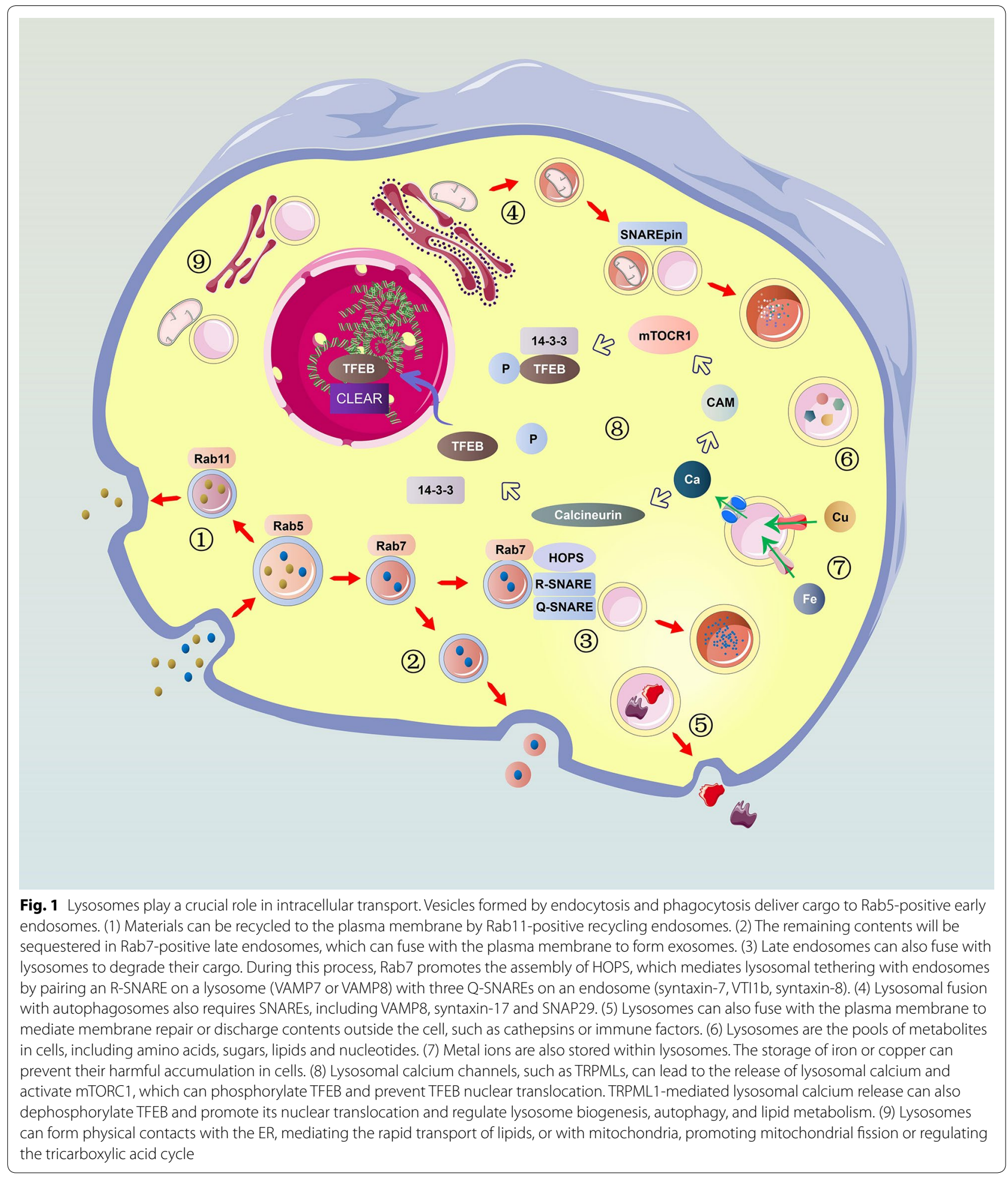

formed during this process transport their contents to early endosomes, which are Rab5 positive. Then, early endosomes become Rab7 positive. During this conversion, internalized materials can be recycled back to the plasma membrane in recycling endosomes, which are Rab11 positive. Meanwhile, the remaining contents can be stored in intraluminal vesicles (ILVs) in late endosomes, which are also termed multivesicular 


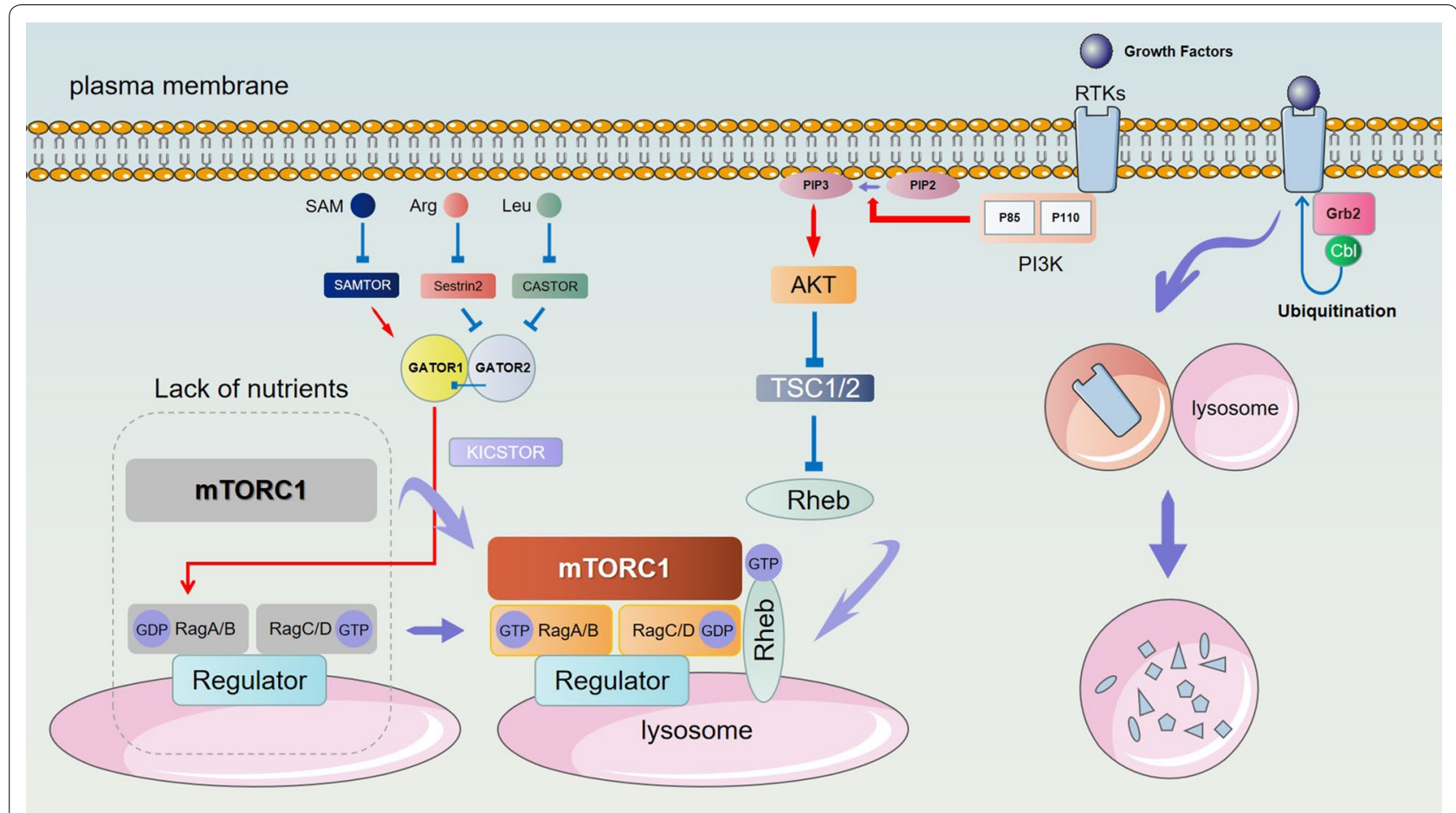

Fig. 2 Lysosomes function as an intracellular signal transduction platform. The combination of growth factors and RTKs can activate the PI3K/AKT signaling pathway and negatively regulate TSC1/2, promoting Rheb to become GTP loaded, which can activate mTORC1. Rag GTPases are localized to lysosomes by Ragulator. When nutrients are lacking, mTORC1 is inactive in the cytoplasm, RagA/B is GDP loaded, RagC/D is GTP loaded, and Rag GTPases cannot bind to mTORC1. GATOR1 is a GAP for RagA/B, and its activity can be antagonized by GATOR2. Sestrin, CASTOR, and SAMTOR can sense Leu, Arg and SAM and interact with GATOR1/GATOR2. KICSTOR mediates GATOR1 recruitment to lysosomes and allows RagA/B to become GTP loaded and bind to mTORC1. Then, GTP-loaded Rheb unlocks mTORC1 kinase activity at the lysosome. Moreover, ligands binding to RTKs (e.g., EGFR) can recruit Grb2, which binds to Cbl. RTKs can be ubiquitinated by Cbl and trafficked to lysosomes for degradation

bodies (MVBs) $[2,3]$. MVBs can move to the plus end of microtubules, mediated by kinesin, and fuse with the plasma membrane to form exosomes and secrete their ILVs. Alternatively, MVBs can move to the minus end of microtubules, mediated by dynein, and fuse with lysosomes to degrade their cargo. Many factors participate in the fusion of MVBs and primary lysosomes [4]. Small GTPases of the Rab family, particularly Rab7, can promote the assembly of tethering complexes (CORVET, HOPS), which mediate lysosomal tethering with endosomes. Tethering is followed by pairing of an R-SNARE (soluble N-ethylmaleimide-sensitive fusion attachment protein receptor) on the lysosomal membrane (VAMP7 or VAMP8) with three Q-SNAREs on the target membrane (syntaxin-7, VTI1b, and syntaxin-8). The four SNAREs, also termed SNAREpin, can bring the two bilayers sufficiently close for fusion to occur. Lysosomes can also discharge their contents outside the cell or mediate membrane repair by fusing with the plasma membrane [5, 6].

Moreover, lysosomes play an important role in autophagy. In mammalian cells, autophagy stimulation signals such as starvation can activate ULK complex (ULK1/2, FIP200, Atg13), which marks the initiation of formation of the phagophore. The recruitment of PI3K complex (VPS34, VPS15, Beclin1 and Atg14) follows, generating the endoplasmic reticulum domains called omegasomes, which contain PI3P. This process requires the participation of membranous vesicles containing Atg9. Omegasomes are highly curved regions and form autophagsomes by membrane expansion. This process requires the participation of two 2 ubiqutinlike conjugation complexes: Atg12-Atg5-Atg16L and LC3 [7]. After closure of the phagophores, the doublemembrane autophagosomes mature and fuse with lysosomes. Under the mediation of dynein, kinesin, and MYO6, the autophagosomes and lysosomes move closer together [8]. Various molecules are involved in the fusion of autophagosomes and lysosomes, mainly including the following: the HOPS complex, Rab7, and adaptors that link autophagosomal or lysosomal components. The HOPS complex can interacts with autophagosomal Q-SNARE STX17 with the help of Atg14 [9]. Rab7 binds HOPS complex and promotes tethering [10]. The adaptor 
proteins, such as EPG5, TECPR1, and PLEKHM1, can bind to autophagosomal or lysosomal components, such as LC3 and ATG12-ATG5, while also interacting with the SNARE complex and/or core tethering factors (RAB7, the HOPS complex), thus ensuring the specificity of fusion [8]. The core machinery for fusion: SNAREs. A trans-SNARE complex, composed of autophagosomal STX17, SNAP29 and lysosomal R-SNARE VAMP8, can mediates the fusion of autophagosomes and lysosomes [11]. Upon fusion of lysosomes with the outer membrane of autophagosomes, the lysosomal contents enter the space between the two autophagosome membranes, and degradate the inner membrane in an LC3-dependent manner. Once autophagy is terminated, lysosomal membrane proteins are recycled from autolysosomes through tubular structures. At the tips of these tubules, nascent lysosomes, also named proto-lysosomes, are formed through a scission/budding process with the participation of KIF5B and DNM2 (dynamin 2) [8].

Lysosomes are pools of metabolites in cells that include amino acids, sugars, lipids and nucleotides. Metal ions are also stored within the lumen of lysosomes. Iron and copper storage within the lysosome can prevent their harmful accumulation in cells $[12,13]$. Transient receptor potential mucolipin (TRPML) channels and two-pore channels (TPCs) are lysosomal calcium channels that can affect the functional status of lysosomes by regulating lysosomal calcium homeostasis; they also play important roles in regulating many other cellular processes [14]. The activation of TRPML1 leads to the release of lysosomal calcium, which can induce the association of calmodulin (CaM) with mTOR; this in turn activates the mammalian target of rapamycin complex 1 (mTORC1). The activation of mTORC1 can result in the phosphorylation of transcription factor EB (TFEB) and promote its interaction with 14-3-3, which then prevents TFEB nuclear translocation. Interestingly, TRPML1-mediated lysosomal calcium release can dephosphorylate TFEB and promote its nuclear translocation. TFEB can bind to the coordinated lysosomal expression and regulation (CLEAR) motif and regulate lysosome biogenesis, autophagy, and lipid metabolism. The activation of TPCs can also lead to TFEB dephosphorylation, which promotes its nuclear translocation. Furthermore, mTORC1 activation can block TPC activity [15].

Moreover, lysosomes can engage in physical contact with other organelles, such as the ER and mitochondria, by means other than membrane fusion. Within these contacts, the bilayers are maintained in close proximity by specialized tethering proteins. Some tethering proteins harbor specialized lipid-binding domains that can mediate the rapid transport of lipids [16, 17]. Contacts between lysosomes and mitochondria can promote mitochondrial fission or transport lysosome-derived metabolites into the mitochondrial matrix to fuel the tricarboxylic acid cycle [18-21].

\section{Lysosomes function as an intracellular signal transduction platform}

Lysosomes play a crucial role in sensing nutrients and regulating cell proliferation and growth. When growth factors bind to receptor tyrosine kinases (RTKs), the $\mathrm{PI} 3 \mathrm{~K} / \mathrm{AKT}$ signaling pathway is activated and negatively regulates TSC1/2 Rheb GAP (GTPase-activating protein), which promotes the GTP-loading of Rheb. When GTP-loaded Rheb is recruited to lysosomes, it will promote the activation of mTORC1 [22, 23]. Rag GTPases are anchored to the lysosomal membrane by Ragulator and V-ATPase proteins. When nutrients are lacking, mTORC1 is inactive and located in the cytoplasm; RagA/B is GDP loaded and $\operatorname{RagC/D}$ is GTP loaded, and thus, the Rag GTPases cannot bind to mTORC1 [24]. The GATOR1 complex is a GAP for RagA/B whose activity can be antagonized by GATOR2 [25]. In response to nutrients, mTORC1 is recruited to the lysosomal membrane. Nutrient sensors, such as Sestrin, CASTOR, SAMTOR and SLC38A9, can sense their ligands, leucine, arginine, $\mathrm{S}$-adenosylmethionine and other amino acids in the lysosomal lumen and interact with the GATOR1/GATOR2 complex. Then, KICSTOR mediates GATOR recruitment to lysosomes and allows RagA/B to become GTP loaded [26]. Nutrient signals converge on Rag GTPases, which physically recruit mTORC1 to lysosomes, while growth factor signals converge on Rheb GTPase, which allosterically unlocks mTORC1 kinase activity at the lysosome [27]. The activation of lysosomal mTORC1 signaling can direct cell metabolism towards growth or promote quiescence and repair and inhibit the formation of autophagosomes. As mentioned above, mTORC1 also participates in the process of lysosomal calcium channel regulation of TFEB nuclear translocation.

Lysosomes can affect growth factor signaling through the endocytic degradation of growth factors, their receptors, or their signal transduction mediators [28]. Lysosomal degradation of RTKs plays an important role in regulating proliferation. Ligands that bind to epidermal growth factor receptor (EGFR) can recruit the adaptor protein Grb2, which binds to Cbl E3 ubiquitin ligase. EGFR is ubiquitinated by $\mathrm{Cbl}$ and then trafficked to the lysosome for degradation [28, 29]. Ligand binding can also recruit p70 and Clip4, which can interact with ubiquitinated EGFR to prevent lysosomal degradation. The same process can also control platelet-derived growth factor receptor (PDGFR) levels [30]. 


\section{Role of lysosomes in human disease}

Lysosomes had been found to be involved in human diseases even before they were recognized as independent organelles. Mutations in genes encoding lysosomalrelated proteins result in a family of diseases termed as lysosomal storage disorders (LSDs) [31]. LSDs are rare diseases, mostly inherited in an autosomal recessive manner. At present, more than 50 types of LSDs are known, and their pathogenesis is not yet fully understood. LSDs are characterized by the accumulation of digested products in the lysosomal lumen, such as amino acids, lipids, sugars and nucleotides [31]. The abnormal accumulation of digestion products in the lysosomal lumen will progressively disrupt its basic functions, including trafficking and the ability to fuse with autophagosomes, which in turn lead to neurodegeneration, metabolic imbalance and severe growth retardation [32, 33]. LSDs are usually classified according to their accumulated substrates and include sphingolipidoses, oligosaccharidoses, mucolipidoses, mucopolysaccharidosis, lipoprotein storage disorders, lysosomal transport defects, neuronal ceroid lipofuscinoses and other types. In addition, lysosomal dysfunction has been shown to be related to the pathogenesis and progression of Parkinson's disease, Huntington's disease and Alzheimer's disease, in which damage to the autophagy-lysosome system leads to impaired cell viability [32].

In recent years, much attention has been paid to the relationship between lysosomes and cancer. In addition to the degradation of substances, lysosomes are proposed to be involved in numerous cell biological processes, including intracellular pathogen destruction, plasma membrane repair, antigen presentation, cell adhesion/ migration, apoptosis, metabolic signal transduction, exosome release, and gene expression regulation [1, 34].

These processes are all closely related to cancer development and progression. Existing studies have shown that lysosomes help cancer cells cope with environmental stress and participate in cancer development and progression mainly through the following aspects. (1) Lysosomes directly degrade nonessential macromolecules through the autophagy pathway [35]. During cancer development and progression, the demand for nutrients and energy by cancer cells increases. In the case of local ischemia and hypoxia, the autophagy pathway in cancer cells is activated to cope with a lack of sufficient nutrients. The hydrolases in lysosomes degrade various intracellular and extracellular macromolecules to recycle and reuse biological materials [36-38]. (2) Lysosomes regulate, transport and degrade cell surface molecules, such as cytotoxic glycoprotein $\mathrm{T}$ lymphocyte antigen-4 (CTLA-4), thus participating in the immune regulation of cancers [37, 39, 40]. (3)
Lysosomes regulate intracellular and extracellular $\mathrm{pH}$ to ensure cancer cell survival [41, 42]. Due to aerobic glycolysis, the production of lactic acid in cancer cells is increased, resulting in an increase in the intracellular $\mathrm{H}^{+}$concentration. The resulting acidic microenvironment seriously affects the activity of cancer cells. Proton pumps on the lysosomal membrane transport $\mathrm{H}^{+}$ into the lysosome lumen, thereby stabilizing the $\mathrm{pH}$ in the cytoplasm of cancer cells [41, 42]. (4) Lysosomes release cathepsin and "remodel" the extracellular matrix (ECM) [40, 43, 44]. Lysosomes can move to the plasma membrane, release various enzymes, such as cathepsin, to the outside of the cell and degrade collagen fibers and integrins to "remodel" the ECM and promote the metastasis of cancer cells $[40,43,44]$. (5) In cancer cells, MVBs can move to the plus end of microtubules and fuse with the plasma membrane to form exosomes and secrete their ILVs. Hence, exosomes are currently recognized as important mediators of cell-tocell communication in cancer progression and metastasis [45]. It has been proposed that let-7 miRNAs play a tumor-suppressive role in targeting oncogenes such as RAS and that cancer cells can release let-7 miRNAs via exosomes to maintain their oncogenicity $[46,47]$.

Further studies have shown that the activity of multiple enzymes in lysosomes is significantly increased in many cancer tissues compared with paracancerous tissues [48], which suggests that lysosome functions are active. In addition, lysosome functions are associated with their spatial distribution. In cancer cells, the expression and activation of kinesin and dynein, which regulate lysosome movement, change dynamically to regulate the locations of lysosomes within cells. The spatial distribution of lysosomes is involved in cancer cell metastasis and drug resistance $[49,50]$.

Therefore, lysosome functional changes and spatial distribution changes are both closely related to cancer development and progression [36, 51]. Comprehensive examination of specific lysosome changes in cancers and in-depth investigation of the intrinsic molecular mechanisms underlying these changes can yield a more comprehensive understanding of the dynamic changes in carcinogenesis and cancer development, thereby providing more opportunities for cancer diagnosis and targeted lysosomal treatment.

\section{Lysosome functional status and cancer development and progression}

As mentioned above, lysosomes participate in a variety of life activities in normal cells. Likewise, lysosomes play crucial roles in cancer development and progression (Table 1). 
Table 1 Lysosomes play crucial roles in cancer development and progression

\begin{tabular}{lc}
$\begin{array}{l}\text { Lysosome-associated biological processes } \\
\text { or molecules }\end{array}$ & Mechanism \\
\hline Endocytosis & Recycling of exogenous materials provides \\
Macropinocytosis & energy, lipids and amino acids for cancer cells. \\
Phagocytosis & H-rasG12V can stimulate membrane ruffling \\
& and pinocytosis. Macropinocytosis is a feature of \\
& RAS-transformed cells and can provide energy \\
& or metabolite precursors and scavenge lipids \\
& in Kras-driven cancer cells, such as pancreatic \\
Autophagy & cancer and lung adenocarcinoma
\end{tabular}

(Atg5, Atg7, ULK1, BECN1)

mTORC1

Lysosome biogenesis

Hydrolase

Lysosomal peripheral localization

Cathepsins

Cathepsin-activated MMPs

Lysosome-derived exocytosis

Lysosomal membrane proteins (LAMP-1)

V-ATPase protein

\section{Effect on cancer}

Dysregulating cellular energetics

Dysregulating cellular energetics

Dysregulating cellular energetics

Promoting the immortalization of cancer cells

Premalignant cells evade oncogene-induced senescence to replicate indefinitely. Autophagydependent lysosomal processes can process senescence-associated chromatin fragments and maintain senescence-mediated tumor suppression. SV40 transformation, MYC expression, and mutant KRAS expression can increase the expression of cathepsins and glycosidases. Inactivation of p53 results in a lack of cathepsin activation. Lysosomal peripheral localization maintains cell membrane integrity and repair during cancer cell division

Cathepsins (e.g., cathepsins B, S, and E) and cathepsin-activated MMPs can degrade extracellular matrix to promote local invasion. Lysosomederived exocytosis of heparinase and cathepsins changes cell shape to promote invasion by cancer cells. Loss of cathepsin B in a mouse mode of pancreatic cancer may decrease metastasis to the liver. Cathepsin L may also play a role in bone metastasis. LAMP-1 is highly expressed in highly metastatic tumor cells, especially metastatic colon cancer cells, indicating that lysosomal membrane proteins are important in cell adhesion and migration. The V-ATPase protein located in the lysosomal membrane can cause an acidic tumor microenvironment and promote the activity of hydrolases
Activating invasion and metastasis 
Table 1 (continued)

Lysosome-associated biological processes or molecules

Cathepsins

Cathepsin-activated MMPs

VEGFR2 recycling

Immune checkpoint recycling and degradation Exocytosis of secretory lysosome

TRPMLS

Tumor antigen processing and presentation Autophagy

Autophagy

Lysosomal iron
Mechanism

Cathepsins and MMPs can promote angiogenesis by remodeling the extracellular matrix and

basement membrane. The pro-form of cathepsin

D stimulates mitogen-activated protein kinase signaling and angiogenic gene expression. It has been shown that cathepsin $\mathrm{K}$ has a role in neovascularization under hypoxic conditions by activating NOTCH1 signaling. The lysosome also functions in regulating endosome-to-plasma membrane recycling of VEGFR2

Lysosomes play a crucial role in regulating tumor immunity. The expression, recycling, and degradation of immune checkpoints, such as CTLA-4 and PD-1, are dictated largely by lysosomal regulation. Secretory lysosomes can impact the function of NK cells and CTL by releasing granzymes, perforin, chemoattractants and so on. Lysosomal exocytosis can increase the surface area of the phagocytosing macrophage and promote engulfment of large particles. TRPMLs can impact immune function by regulating lysosomal exocytosis, endocytosis, and phagocytosis

Lysosomes play an important role in tumor antigen processing and presentation. The deficiency of autophagy causes p62 (an autophagy adaptor) accumulation in HCC, which results in the generation of ROS through the dampening of NF-KB signaling. P62 accumulation, NF-KB signaling inhibition, and ROS generation can promote tumorigenesis by dampening dendritic cell function and impairing the antitumor immune response

Lysosomes can stimulate programmed cell death by the activation of autophagy or the lysosomal protease-dependent activation of caspases. The defect of lysosomes in cancer cells causes the inability to clear dead cell debris, which leads to the survival of neighboring cells. There is crosstalk between the autophagy and apoptosis pathways in cancer. The antiapoptotic protein BCL-2 can promote cancer cell survival by limiting autophagy and preventing autosis. The accumulation of iron in lysosomes creates favorable conditions for ROS formation by Fenton reactions to promote tumorigenesis

Lysosomal enzymes Glucocorticoid receptor Autophagy (ATG7, Beclin 1)

\section{Effect on cancer}

Promoting angiogenesis

Impairing antitumor immune response

Impairing antitumor immune response

Resisting cell death

Tumor-promoting inflammation
Lysosomes play an important role in tumor initiation stimulated by chronic inflammation. Cathepsin B can cleave trypsinogen-1, cause pancreatitis, and increase pancreatic cancer risk. Heparanase activated in the lysosome can degrade heparin sulfate proteoglycans and thereby regulate the activity of cytokines and growth factors such as TGF- $\beta$. Defects in autophagy genes ATG7 and Beclin 1 also cause chronic inflammation and promote spontaneous cancer of the lung, liver and lymphocytes 
Table 1 (continued)

\begin{tabular}{|c|c|c|}
\hline $\begin{array}{l}\text { Lysosome-associated biological processes } \\
\text { or molecules }\end{array}$ & Mechanism & Effect on cancer \\
\hline $\begin{array}{l}\text { Autophagy (Beclin, Atg5) } \\
\text { Lysosomal integrity }\end{array}$ & $\begin{array}{l}\text { Autophagy and lysosome activity participate in } \\
\text { the maintenance of genome stability. Kidney } \\
\text { cells isolated from Beclin 1+/- Atg5 - / - mice } \\
\text { show accumulation of p62 (an autophagy } \\
\text { adaptor). Serial passage of these cells leads to } \\
\text { alteration of cell ploidy and genomic instability. } \\
\text { Because of deficient autophagic flux, cells can- } \\
\text { not recycle nucleic acids, leading to nucleotide } \\
\text { depletion and DNA damage. Cancer cells are } \\
\text { usually aneuploid. Untransformed aneuploid } \\
\text { cells show decreased autophagy, which cor- } \\
\text { relates with the degree of karyotypic imbalance. } \\
\text { A lack of lysosomal integrity causes leakage } \\
\text { of DNAses from the lysosome and promotes } \\
\text { tumorigenesis }\end{array}$ & Promoting genome instability \\
\hline $\begin{array}{l}\text { RTK recycling and degradation } \\
\text { Autophagy } \\
\text { Lysosome membrane }\end{array}$ & $\begin{array}{l}\text { Lysosomes can regulate proliferative signaling } \\
\text { through the endocytosis, degradation and } \\
\text { recycling of RTKs. Autophagy pathways can } \\
\text { also regulate proliferative signaling through } \\
\text { the uptake and degradation of intracellular RTK } \\
\text { signaling mediators. The activation of mTORC1 } \\
\text { depends on its correct position to the lysosome } \\
\text { membrane surface }\end{array}$ & Sustaining proliferative signaling \\
\hline M6P/IGF2R & $\begin{array}{l}\text { M6P/IGF2R is responsible for the proper trafficking } \\
\text { of lysosomal hydrolase. Lysosomal hydrolase } \\
\text { can be delivered to lysosomes, where signaling } \\
\text { factors are processed that play a role in tumor } \\
\text { suppression. The expression of M6P/IGF2R in } \\
\text { hepatocellular carcinoma is decreased. M6P/ } \\
\text { IGF2R is also mutated in colon, breast, prostate, } \\
\text { kidney, and lung cancers. Mutant M6P/IGF2R } \\
\text { leads to mislocalization of hydrolase and fails to } \\
\text { activate TGF- } \beta \text {, a class of conserved cytokines } \\
\text { that suppress cell proliferation }\end{array}$ & Sustaining proliferative signaling \\
\hline
\end{tabular}

\section{Lysosomes and cancer energy metabolism}

Continuous proliferation requires a sufficient energy supply and raw materials for macromolecular synthesis. The uptake and decomposition of extracellular glycoproteins and glycolipids and the recycling of intracellular substances are pathways for cancer cells to obtain carbohydrates, lipids and amino acids [52]. The extracellular substances obtained by phagocytosis, endocytosis and macropinocytosis can be further delivered to lysosomes to generate nutrients through lysosomal degradation. Moreover, through autophagy, intracellular substances are degraded into the nutrients and energy required by cancer cells. Although the microenvironment of cancer cells is poor, another core function of lysosomes in cancer cells is to provide energy and metabolize precursors through the recycling of endogenous or exogenous macromolecules $[53,54]$. In KRAS-driven lung cancer and pancreatic ductal adenocarcinoma cells, lysosomes can degrade substances that are recycled from the extracellular and intracellular environments to provide materials for cancer cell growth [55], and prevent AMP accumulation, energy crisis, and fatal nucleotide degradation [56]. As mentioned above, lysosomes play a key role in cellular nutrient sensing. Studies have found that some amino acids can be directly sensed and bound by molecules such as amino acid receptors and transporters in the plasma membrane and cytoplasm as signal molecules; these amino acids can also be perceived by lysosomes [54]. mTORC1 is a highly conserved kinase complex in eukaryotic cells that can sense and integrate stimulation information such as energy and nutrient status to regulate cell growth and autophagy. When nutrients are lacking in cancer cells, MiT/TFE family of transcription factors can escape mTORC1-mediated negative regulation and locate in nucleus, thereby allowing cancer cells to maintain the activation of mTOR signaling and autophagy at the same time [57]. The activation of autophagy ensure efficient recycling of cellular material. This mechanism is associated with a variety of cancer metabolic activities. In cancers such as pancreatic ductal adenocarcinoma, renal cell carcinoma and non-small cell lung cancer, TFE3/TFEB and other transcription factors 
are activated to promote lysosomal biogenesis and functional activation, thereby maintaining steady-state metabolism in cancer cells and further promoting cancer malignancy [58-60]. This signal transduction mechanism not only upregulates lysosome biosynthesis but also increases autophagy to help cells cope with nutritional stress. The deletion of GATOR1 has been observed in human cancers and suggests that aberrant mTORC1 nutrient sensing plays a crucial role in cancers [25].

\section{Lysosomes maintain cancer cell proliferation}

Malignant cells must avoid oncogene-induced senescence (OIS) to achieve continuous proliferation [61]. The role of OIS in the inhibition of carcinogenesis is very important and involves gene expression at cell cycle checkpoints and activation of the aging-related secretory phenotype [62-65]. Interestingly, a large number of lysosomespecific phenotypes can be observed in senescent cells, including upregulated lysosomal gene expression and increased lysosome number/volume [66, 67]. The metabolic activity of senescent cells was originally thought to be lower than that of proliferating cells; however, studies have shown that the metabolism of senescent cells is actually hyperactive and that the corresponding changes in lysosomes may provide a greater material basis for senescent cells [68-71]. During the OIS process, cellular oxidative metabolism increases, which is often associated with changes in chromatin structure, such as senescence-related heterochromatin. Heterochromatin foci can be extruded from the nucleus and enter the cytoplasm. Cancer cells degrade these cytoplasmic chromatin fragments by increasing the level of autophagy through increased lysosome synthesis, thereby maintaining the function of cancer cells and slowing aging [72]. Interestingly, the activation of proto-oncogenes or the absence of tumor suppressor genes can induce cell proliferation and induce changes in lysosome synthesis. In SV40-mediated immortalized transformed cells, molecular events such as MYC gene amplification and overexpression and KRAS mutant expression can increase the expression of lysosome catalase and glycosidase (including cathepsin $\mathrm{D}$ and cathepsin E) [73], suggesting that the expression of oncogenes can increase the number of lysosomes and enhance their functional state. In KrasG12D-driven lung tumor cell, the deletion of Atg5 or Atg7 reduces cell proliferation and tumor burden, suggesting that this is due to impaired autophagy. Atg7 deficiency can activate p53, which contributes to tumor suppression [74, 75]. Atg7 deficiency also reduces intiation, proliferation and development of melanoma, prostate cancer and colorectal cancer [76-78]. FIP200 is an essential autophagy protein to initiate autophagosome formation and the ablation of FIP200 can diminish the tumor-initiating properties of breast cancer stem cells [79]. Lysosomal calcium homeostasis can affect tumor proliferation. TRPML-2 knockdown can inhibit cell viability and proliferation, affect the cell cycle, promote apoptotic cell death in glioma cell lines. The mRNA and protein levels of TRPML-2 have been shown to increase with pathological grade [80]. The above results indicate that during the process of unregulated cancer cell proliferation, lysosomes exhibit increased biosynthesis and an enhanced functional status, which promotes intracellular substance circulation and the degradation of harmful intracellular byproducts, thereby maintaining cancer cell proliferation.

\section{Lysosomes promote cancer invasion and metastasis}

Invasion and metastasis are the most prominent biological characteristics of malignant cancers and are also the leading causes of death among patients. Epithelial-mesenchymal transition (EMT) plays a critical role in cancer metastasis by enabling epithelial cells to acquire motility and invasiveness, which are characteristic of mesenchymal cells [81]. Autophagy plays an important role in cancer invasion and metastasis. Studies have found that autophagy is activated under adverse conditions, such as hypoxia and the accumulation of acidic metabolic products. Cells can use autophagy to degrade epithelialderived molecules such as E-cadherin to induce EMT, thereby enhancing cancer cell invasiveness and metastasis [82, 83]. In vitro, EMT-inducing factors can downregulate the expression of E-cadherin on the plasma membrane of cancer cells by promoting the degradation of E-cadherin in lysosomes and inhibiting recycling, which suggests that the lysosomal degradation pathway promotes invasion and metastasis [84]. In addition, some metastasis suppressors, such as NM23-H1, can promote breast cancer invasion through lysosomal degradation [85]. Autophagy also supports cancer invasion and metastasis by promoting disassembly of cell-matrix FAs. This process was mediated by the interaction of processed LC3 with paxillin, a key FA component [86]. Autophagy-dependent secretion of the proinvasive cytokine, such as IL6, also promotes cancer invasion [87].

Degradation and modification of the ECM are necessary conditions for cancer invasion and metastasis [88, 89]. The release of lysosomal hydrolases, such as cathepsin, plays an important role in this process. TRPMLs and TPCs can affect the functional status of lysosomes and promote tumor invasion and metastasis by regulating lysosomal calcium homeostasis [14]. TRPML1mediated lysosomal calcium release can promote TFEB nuclear translocation and increase lysosome biogenesis and autophagy. The activation of TPCs also promotes TFEB nuclear translocation. In the human hepatocellular carcinoma cell line HepG2, tetrabromobisphenol A 
(TBBPA) activates TRPML1, which promotes the release of lysosomal calcium and the nuclear translocation of TFEB and increases lysosomal exocytosis. Cancer cells then secrete cathepsins through lysosomal exocytosis [15]. Cathepsin can act directly or through the activation of matrix metalloproteinases (MMPs) to degrade and remodel the ECM, thus enhancing the invasion and metastasis of cancer cells. A study on a mouse model of pancreatic cancer found that the absence of cathepsin B reduced the probability of liver metastasis and prolonged the survival time of cancer-bearing mice [90]. Cathepsins $\mathrm{B}, \mathrm{S}$, and $\mathrm{E}$ are all involved in invasion and metastasis in various cancers [91-93]. Silencing TPC1 and TPC2 can reduce the adhesion and migration of invasive tumor cells. The inhibition of TPCs leads to the accumulation of integrins in endocytic vesicles and to impaired formation of leading edges. Alternatively, the inhibition of TRPMLs or TPCs may affect EGFR recycling and possibly delay or prevent cancer cell migration and/or proliferation [94, 95]. In addition, studies have confirmed that various lysosomal proteins, such as lysosome-associated protein-1 (LAMP1) [96, 97], LAMP3 [98, 99] and LAPTM4BP [100], are highly expressed in many malignant cancers, including melanoma, lung cancer, breast cancer and liver cancer, and that such high expression is associated with invasion and metastasis. LAMP-1 is abundant on the cell surface of highly metastatic cancer cells, especially metastatic colon cancer cells, which suggests that lysosomal proteins are important in cell adhesion and migration [101]. Researchers have examined the sensitivity of bladder cancer cell lines with different invasive potentials to the lysosomal inhibitors chloroquine $(\mathrm{Cq})$ and bafilomycin and found that highly invasive bladder cancer cells were more sensitive to $\mathrm{Cq}$ and bafilomycin, while the invasive ability of Cq-resistant cells selected by screening highly invasive cells was significantly decreased [102]. These results suggest that lysosomes can be used as potential therapeutic targets in metastatic cancers.

\section{Lysosomes promote cancer angiogenesis}

Angiogenesis has an important impact on cancer growth, invasion and metastasis. Remodeling of the ECM and vascular basement membrane is essential for initiating angiogenesis and vascular sprouting $[103,104]$. The lytic granules cleaved by lysosomal exocytosis can destroy vascular basement membrane components at physiological $\mathrm{pH}$ [105]. Studies have shown that cathepsins $\mathrm{D}, \mathrm{B}, \mathrm{S}, \mathrm{K}$ and $\mathrm{L}$ all play roles in promoting angiogenesis. On the one hand, activation of MMPs by cathepsin can mimic angiogenesis; on the other hand, cathepsin can directly act as a cytokine to stimulate the proliferation of vascular endothelial cells, thereby playing a role in promoting angiogenesis [106]. In addition, under anoxic conditions, cathepsin $\mathrm{K}$ can play important roles in angiogenesis through the activation of Notch homolog 1 , translocation-associated (NOTCH1) signaling. Cathepsin $\mathrm{K}$ knockdown in endothelial cells results in reduced angiogenesis [107]. In addition, lysosomes also play a role in endothelial cell migration factor regulation. Rab GTPase is essential for angiogenesis and participates in the endosomal recycling of vascular endothelial growth factor receptor 2 (VEGFR2) [108]. Genetic deletion of Rab4a and Rab11a and the inhibition of lysosome activity by chloroquine can lead to defects in $\mathrm{VCl} 2$ lysosomalplasma membrane recycling and inhibition of endothelial cell migration [108]. Lysosomal calcium homeostasis is associated with angiogenesis. The blockade of TPCs can inhibit VEGF-induced neoangiogenesis, which is mediated by TPC2-dependent calcium signaling. The inhibition of signaling pathways involving VEGFR2, NAADP, $\mathrm{TPC} 2$, and $\mathrm{Ca} 2$ + release from acidic stores can greatly reduce the activation of VEGFR2 downstream targets, which would then block angiogenesis, in both in vitro and in vivo models [109]. In-depth studies on the internal mechanisms and key molecules of lysosomal-regulated angiogenesis are currently lacking. Further studies on the specific roles of lysosomes in cancer angiogenesis may lead to the development of new anti-angiogenesis therapeutic strategies.

\section{Lysosomes and cancer immunity}

In recent years, the great success of immune checkpoint therapy has confirmed the role of the immune system in cancer treatment [110]. It has been shown that lysosomes can serve as a major destruction location for immune checkpoint molecules, as secretory lysosomes can temporarily store immune checkpoint proteins, such as CTLA-4, PD-L1, TIM-3, CD70, CD200, and CD47 [111]. Studies have shown that CTLA-4 is a transmembrane $\mathrm{T}$ cell inhibitory protein mainly located in the plasma membrane and cytoplasm; however, attachment to the plasma membrane is important for CTLA-4 to perform its functions [112]. CTLA-4 expression is largely regulated by lysosomes. On the one hand, lysosomes degrade CTLA-4; on the other hand, lysosomes are responsible for CTLA- 4 transport to the plasma membrane. CTLA-4 can bind to activator protein 1 (AP1) and AP2 [113, 114] to promote its transport to lysosomes for degradation. In addition, CTLA-4 can enter the cytoplasm for lysosomal degradation via endocytosis $[115,116]$. Lysosomes containing CTLA-4 can be transferred to the T cell receptor (TCR), which subsequently secretes CTLA-4, increasing cell surface CTLA-4. After tyrosine phosphorylation, CTLA-4 remains on the cell surface $[117,118]$. Therefore, the expression of other inhibitory receptors in $\mathrm{T}$ cells (e.g., PD-1) can be confidently assumed to also be 
similarly regulated by lysosomes; however, the role of lysosomes in this process is still unclear.

Secretory lysosomes, also known as lytic granules, contain proapoptotic granzymes and perforin and can also participate in the regulation of immune cell functions. Natural killer (NK) cells and cytotoxic T lymphocytes (CTLs) play a crucial role in immunity, as they are responsible for the elimination of both virally infected and tumorigenic cells. The clearance of target cells is dependent on the regulated exocytosis of secretory lysosomes, which can deliver proapoptotic granzymes and perforin to target cells [119]. Upon recognition of target cells, microtubules and actin filaments in CTLs are reorganized, which results in the polarization of the centrosome towards the immunological synapse (IS), which are formed with the target cells. Rab7 can interact with Rab7-interacting lysosomal protein (RILP) to recruit dynein to secretory lysosomes, which mediate minus-end-directed movement of secretory lysosomes to IS. Then, the contents in secretory lysosomes can be released, which leads to the destroy of target cells [120]. However, cancer cell autophagy may serve to intercept granzymes and perforin released by cytotoxic immune cells, blunting the efficacy of anti-tumor immune response [121, 122]. Impaired autophagy in breast cancer cells activates the immune response, IFN production and lymphocyte infiltration [79].

TRPMLs also play an important role in immunity [123]. When macrophages bind particles, the TRPML1 channel in lysosomes becomes activated and mediates $\mathrm{Ca} 2+$ release from lysosomes, which induces lysosomal exocytosis at the site of the phagocytic cup; this in turn increases the surface area of the phagocytosing macrophage and promotes the engulfment of large particles. TRPML1-mediated Ca2+release is indispensable for phagosome maturation [124]. Macrophages can produce and secrete a variety of cytokines and chemokines after stimulation. Tumor-associated macrophages can be stimulated by IL-4, IL-10, or IL-13 and then migrate into tumor tissue, where they perform protumorigenic functions [125]. Recent findings have shown that the TRPML2 channel plays a crucial role in the release of chemokines as well as in the stimulation of macrophage migration [126, 127]. NK cell activity is regulated by the dynamic balance between activating and inhibitory signals, which determine whether NK cells kill the target cell. Major histocompatibility complex (MHC) class I molecules can be recognized by inhibitory receptors. The expression of MHC class I on virus-infected cells and tumor cells is decreased, which will be recognized by NK cells and promote the killing of these cells by NK cells. A process termed NK cell education describes the interaction between self-MHC and inhibitory receptors on NK cells, which calibrates NK cell effector capacities. TRPML1 participates in this process by regulating secretory lysosomes, granzyme B content, and the effector function of NK cells [128].

In summary, lysosomes in cancer cells are involved in various biological events affecting the development and progression of cancers. This finding provides useful clues for the diagnosis and treatment of cancers. Identification of the specific functions of lysosomes can help predict the prognosis of cancer patients and formulate individualized treatments. The functional status of lysosomes is closely related to their intracellular distribution. Understanding and exploring the lysosome distribution in cancer cells and the effects of different distributions on the development and progression of cancers can provide more comprehensive lysosome information and thus a theoretical basis for further individualized diagnosis and treatment strategies for cancer.

\section{The spatial distribution of lysosomes and cancer development and progression}

Lysosomes exhibit different spatial distributions in cancer cells. In most cases, lysosomes are scattered in the cytoplasm, but some lysosomes are concentrated around the nucleus and can also be distributed in the plasma membrane. Studies have shown that in addition to the aforementioned functional status of lysosomes, the spatial distribution of lysosomes significantly affects the biological properties of cancer cells.

\section{Movement and spatial distribution of lysosomes}

Lysosomes are widely distributed in the cytoplasm. In nonpolarized cells, lysosomes are mainly concentrated in the microtubule-organizing center (MTOC) around the nucleus, but some peripheral lysosomes can reach or protrude through the plasma membrane [129]. In cells with obvious polarity, such as neuronal cells, lysosomes are distributed in various parts of the cytoplasm, including soma, axons and dendrites [130]. Lysosomes in the cytoplasm can move along the microtubules in and around the cell center. Movement towards the plus (centrifugal) and minus (centripetal) ends of microtubules is mediated by kinesin [131] and dynein [132], respectively (Fig. 3). Of course, this type of movement does not occur randomly and is often induced and regulated by specific conditions. For example, acidification of the cytoplasm leads to proliferation of the perinuclear lysosome population, and subsequent alkalization can promote the return of these lysosomes to a central location [133, 134]. Other stimuli, such as starvation and drug-induced apoptosis, can cause centripetal aggregation of lysosomes [134, 135].

In nonpolarized cells, the plus ends of microtubules are oriented towards the periphery of cells. In polarized cells 


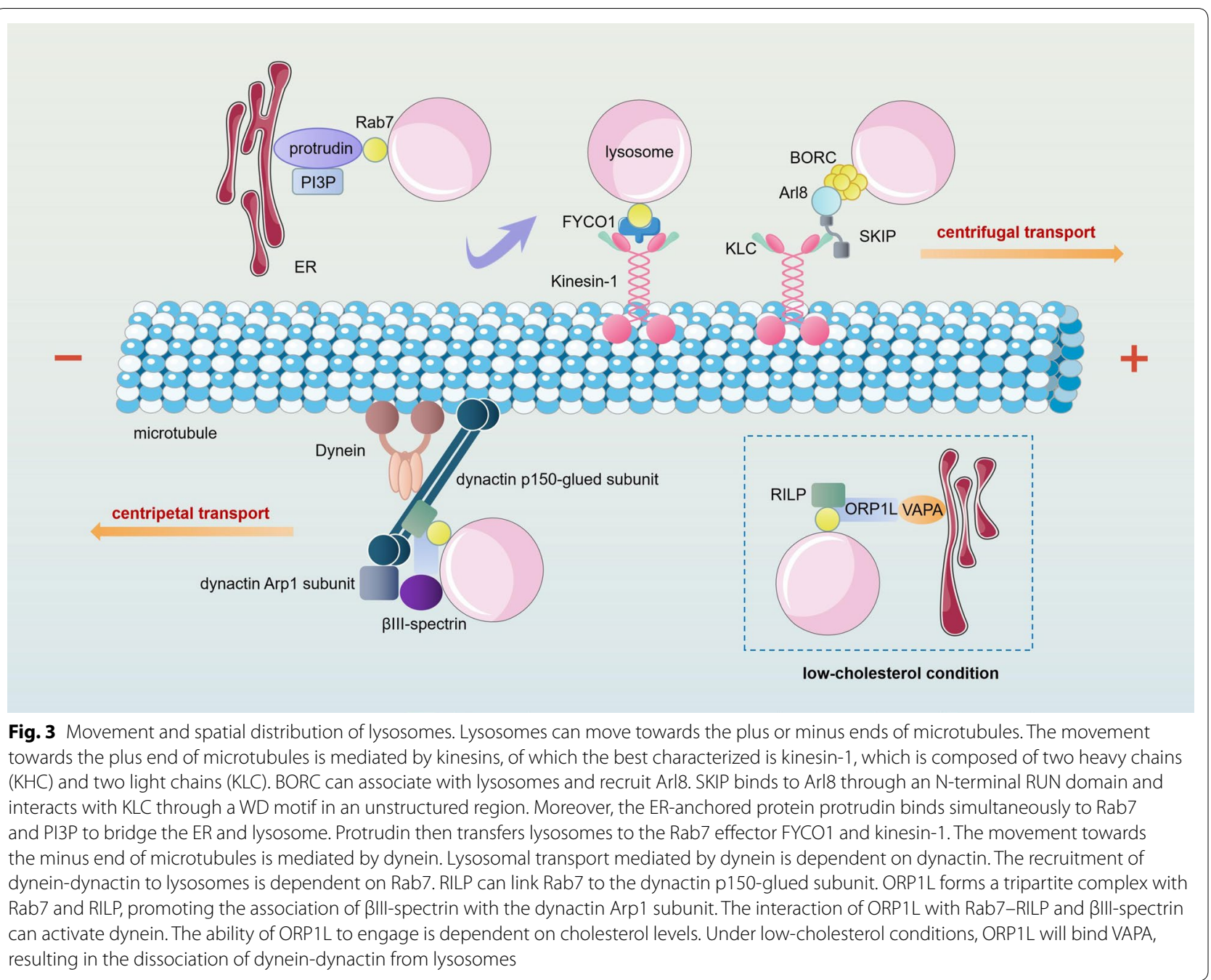

such as neurons, the plus ends are oriented towards the ends of axons. Therefore, in these cells, lysosome transport from the cell center to the periphery (centrifugal transport) is mediated by the kinesins. However, in some polarized cells, such as the dendrites of neurons (microtubules with mixed orientations), microtubules can orient towards other directions [136, 137]. In these cases, both centrifugal and centripetal transports are mediated by the kinesisns. Lysosome movement towards the plus end of microtubules is mediated by the kinesin superfamily (KIF), including kinesin superfamily 1 (KIF5A, KIF5B and KIF5C) [138-140], kinesin superfamily 2 (KIF3) [141, 142], kinesin superfamily 3 (KIF1A and KIF1B) [143$145]$ and kinesin superfamily 13 (KIF2) [143, 146]. Kine$\sin -1$ is the well-characterized kinesin involved in the transport of lysosom. Kinesin-1 is a heterotetramer composed of two heavy chains (KHC) and two light chains (KLC) that can be recruited to lysosomes by the multisubunit BLOC-1-related complex (BORC), the Arf-like small GTPase Arl8, the Arl8 effector SifA, and kinesininteracting protein (SKIP) $[138,147,148]$. BORC is an octameric complex that can associate with the cytosolic face of lysosomes and recruit Arl8 [149]. Then, through an N-terminal RUN domain, SKIP binds to Arl8 and interacts with the tetratricopeptide repeat (TPR) domain of the KLC by a WD motif in an unstructured region. This process completes the linkage of lysosomes to kinesin-1 [138]. The coupling of late endosomes to kinesin-1 involves an alternative mechanism. The Rab7 and phosphatidylinositol 3-phosphate bind to ER-anchored protein protrudin to bridge the lysosome and ER. Protrudin then transfers lysosomes to the Rab7 effector FYCO1 and kinesin-1 [150]. Microtubule-associated proteins (MAPs) usually play inhibitory roles in lysosome movement. A representative example is that the central nervous system TAU proteins can inhibit kinesin superfamily 1-dependent lysosomal transport [151, 152]. However, other MAPs, such as ensconsin and DCLK1, may promote the 
recruitment and activation of kinesins to specific populations of microtubules [153, 154]. In addition, the activity of kinesin can be regulated by kinesin binding protein (KBP). Under the action of KBP, kinesin motor domain inactivation may occur [155].

Lysosomal movement can also occur towards the minus end of a microtubule, which is mediated by dynein. Currently, two types of dynein are believed to exist: axonemal dynein, which has a role in flagella and cilia, and cytoplasmic dynein, which functions in other types of cells. Cytoplasmic dynein is involved in lysosome movement and can move lysosomes from the plus end to the minus end of microtubules (centripetal). In nonpolarized cells, cytoplasmic dynein mediates lysosome transport from the periphery to the center (centripetal) [156]. In neurons, cytoplasmic dynein mediates centripetal lysosome transport, and due to the mixed directionality of dendritic microtubules, cytoplasmic dynein may simultaneously mediate centripetal and centrifugal lysosomal transport in dendrites [157]. Lysosomal transport mediated by cytoplasmic dynein is dependent on dynactin. The recruitment of dynein-dynactin to lysosomes is mainly mediated by Rab7. Rab7-interacting lysosomal protein (RILP) can link Rab7 to dynein-dynactin through the dynactin p150-glued subunit [158]. The oxysterol-binding protein-related protein $1 \mathrm{~L}$ (ORP1L) forms a tripartite complex with Rab7 and RILP, which promotes the association of BIII-spectrin with the dynactin Arp1 subunit [158]. The interaction of ORP1L with Rab7-RILP and BIII-spectrin can activate dynein, and the ability of ORP1L to engage in this process is dependent on cholesterol levels [159]. Under low-cholesterol conditions, the OSBP-related domain of ORP1L will bind the vesicle-associated membrane protein-associated protein A (VAPA), which results in the dissociation of dynein-dynactin from lysosomes and causes dispersal of lysosomes to the cell periphery. In contrast, ORP1L does not interact with VAPA, and dynein-dynactin mediates the movement of lysosomes towards the cell center under high-cholesterol conditions. In addition, other Rab proteins, such as Rab9A, Rab34 and Rab36, the lysosomal Ca2+- sensors ALG-2, LAMP-1, and LAMP-2 and the transmembrane protein 106B (TMEM106B), are also involved in regulating the coupling of lysosomes to dynein-dynactin [36, 160-163].

Generally, intracellular substances do not move in a straight line, as transport routes are usually bidirectional and can change. These dynamics have been observed for mitochondria, peroxisomes, endosomes, lipid droplets, synaptic vesicle precursors, and viral particles [164]. Because kinesin and dynein are unidirectional motor proteins, the above dynamics suggest that kinesin and dynein play a synergistic rather than independent role in intracellular transport. In the axonal transport of vesicles containing the cellular mammalian prion protein (PrPC), kinesin-1 and cytoplasmic dynein can associate with PrPC vesicles to direct bidirectional movement. Kinesin-1 light chains (KLC1), kinesin-1 heavy chains (Kinesin-1C), and dynein heavy chain (DHC1) play a major role in this process. In addition to mediating anterograde transport, KLC1 and Kinesin-1C also activate retrograde movement of PrPC vesicles. The absence of KLC1 or Kinesin-1C results in bidirectional decreases and reduces velocity distributions in both directions. Similarly, decreasing DHC1 can also result in bidirectional movement inhibition. The above phenomenon shows that the activities of these motors are tightly coupled. Despite this, kinesin-1C and KLC1 are not required for the vesicle association of $\mathrm{DHC} 1$, indicating that a stable motor subunit population on vesicles is not affected by the presence or absence of other motors. Motor subunits are associated with stationary and moving PrPC vesicles. Thus, impairment of the reverse movement observed after removing a certain motor subunit is likely a result of coordination between Kinesin-1C and DHC1 activities, instead of resulting from structural changes to motorcargo associations [164].

\section{The effect of the spatial distribution of lysosomes on cancers}

Kinesins such as KIF11, KIF25 and KIF5b are overexpressed in most cancer cells [49, 165]. As mentioned above, these proteins can interact with microtubules to change lysosome localization. When these proteins are knocked down, cancer cells may exhibit cathepsindependent death and increased sensitivity to anticancer drugs such as siramesine [36]. These results suggest that kinesin-mediated changes in lysosomal distribution play an important role in ensuring cancer life activities and drug resistance.

Autophagy is a conserved and multipathway-mediated process for organelle degradation in eukaryotic cells [36]. Autophagy is a type of reactive cellular response to changes in the internal and external environment that plays an important role in cell survival and homeostasis [36]. First, an autophagosome is formed by wrapping proteins, organelles and other molecular particles under the membrane structure. Then, in the process of dyneinmediated centripetal lysosomal transport, lysosomes and autophagosomes fuse to form autophagic lysosomes, thereby completing the autophagy process [36]. Cardoso et al. found that autophagy relies heavily on lysosome distribution. Downregulation of KIF5b in human HeLa cervical cancer cells induces lysosome accumulation around the plasma membrane and autophagosome accumulation around the nucleus and suppresses the fusion 
of autophagosomes and lysosomes. These results suggest that lysosome movement and distribution regulate cancer cell autophagy [49].

The function of immune cells in cancer tissues also depends on the distribution of lysosomes. Immune cells such as mononuclear macrophages can recognize and phagocytose endogenous and exogenous substances and further degrade endocytosed substances in lysosomes. Studies have shown that changes in lysosomal distribution and movement affect the immune killing effect and degradation capacity of mononuclear macrophages [166]. In addition, lysosomes perform an important role in antigen presentation. In mature dendritic cells, antigenic peptides that bind to MHC-II molecules are released from lysosomes, and then are transported to the plasma membrane via lysosomal movements, where they are presented to CD4-positive $\mathrm{T}$ lymphocytes and activate $\mathrm{T}$ cells to perform the corresponding immune functions $[167,168]$. In addition, the lysosomal distribution is closely related to the ability of CTLs and NK cells to clear viruses or cancer cells. CTLs form immune synapses and release the contents of lytic granules into their target cells. Not only specific cytotoxic mediators but also lysosomal cavities and membrane proteins are contained in the lytic granules; therefore, lytic granules are considered "lysosome-associated organelles" [169] or "secretory lysosomes" [170]. Interestingly, CTLs deliver cytotoxic granules based on the same mechanism as lysosomal localizaition. After binding to their target cells, lytic granules in activated CTLs move towards the microtubuleorganizing center, which is located under the plasma membrane of the immune synapse [171]. This movement is mediated by the Rab7-kinesin complex [120]. The Rab27a-kinesin-1 complex mediates movement from the center of microtubule tissues to the terminals of immune synapses [172]. Interfering in this lytic particle movement can greatly reduce the killing ability of CTLs.

The movement of lysosomes towards the periphery of cells is necessary for cancer growth, invasion and metastasis. During cancer progression, lysosomes undergo dynamic changes in quantity, morphology, intracavitary $\mathrm{pH}$, hydrolase content and intracellular distribution [106, 173]. The most notable change is lysosomal transport from the central to peripheral cytoplasm [174, 175]. This redistribution can be induced by changes in the cancer microenvironment, such as a reduction in $\mathrm{pH}[106$, 176], or changes in the expression of genes that regulate lysosomal localization and movement during carcinogenic transformation processes (e.g., an increase in the KIF5B mRNA level in various cancer tissues). The expression of epidermal growth factor receptor 2 (HER2) in human breast cancer cells has been found to upregulate the expression of KIF5B and promote its binding to lysosomes [49]. In addition, studies have found that compared with stromal cells or benign prostatic hyperplasia tissue, Rab7 mRNA levels in prostate cancer cells are significantly downregulated [177]. Rab7 knockdown can cause lysosome redistribution and enhance the invasive ability of cancer cells. Second, lysosomal centrifugal transport is critical for the extracellular secretion of cancer cells and maintenance of plasma membrane integrity $[178,179]$. The peripheral localization of lysosomes can maintain plasma membrane integrity and repair functions during rapid cell division [180], while hydrolases secreted extracellularly can degrade and remodel the ECM, rendering it more suitable for cancer cell movement and thus promoting cancer cell migration and invasion [181, 182]. During extracellular secretion, the transport of MMPs in lysosomes also contributes to the invasion and metastasis of cancer cells [183, 184]. In addition, after activation, integrins such as $\alpha 5 \beta 1$ can be anchored on the lysosomal membrane, which is mediated by Rab25, and be further transported to the plasma membrane through lysosomal movement, thereby regulating the adhesion and migration of cancer cells [185]. The above findings indicate that lysosomal transport is essential for cancer cell exocytosis, ECM degradation and cell adhesion and migration. Lysosomal localization and movement may be potential targets for cancer diagnosis and treatment [186].

\section{Conclusion and prospects}

In summary, the functional status and distribution of lysosomes affect various malignant biological events in cancer cells and regulate the development and progression of cancers. Therefore, real-time monitoring and determining the functional status of lysosomes may facilitate the development of precise personalized treatment regimens. In addition, because lysosomes participate in mitogen signal transduction and immune escape processes, targeted lysosomal treatment may conceivably slow or inhibit the transformation of precancerous lesions into cancer. Furthermore, the functional status of lysosomes is related to the expression of important immune checkpoint receptors; this knowledge may help improve the effectiveness of cancer immune checkpoint therapy. Lysosomes can also degrade important connective proteins (e.g., E-cadherin) by autophagy and phagocytosis. Therefore, inhibiting the degradation of E-cadherin by lysosomes may be a new strategy for treating cancer metastasis.

Lysosomal distribution and movement are dependent on complex interactions between microtubule motor proteins and actin cytoskeleton structures. Although a series of molecules that regulate such interactions have been discovered, more such molecules may be discovered 
in the future. These molecules may have a very close relationship with cancer development and progression. The following outstanding issues should be addressed in future work: How do lysosomes in cancer cells switch between static and dynamic states and between centrifugal and centripetal movements? What are the roles of different lysosomal movements and different lysosomal distributions in cancers? How are lysosomal movements and distributions regulated in cancer cells through conditions such as nutrient availability, extracellular $\mathrm{pH}$ changes and cellular stress? What effects do lysosomal movement and distribution interventions have on lysosome function in cancer cells? Can cancers be treated by regulating lysosome distribution? Obtaining answers to these questions will not only help to clarify the mechanisms underlying the development and progression of cancers but also provide information for therapeutic interventions for cancers.

\begin{abstract}
Abbreviations
mTORC1: Mammalian target of rapamycin complex 1; CTLA-4: Cytotoxic T lymphocyte antigen 4; TCR: T cell receptor; TFEB: Transcription factor EB; rER: Rough endoplasmic reticulum; sER: Smooth endoplasmic reticulum; ECM: Extracellular matrix; GATOR: GAP activity towards Rags; OIS: Oncogeneinduced senescence; EMT: Epithelial-mesenchymal transition; MMPs: Matrix metalloproteinases; LAMP1: Lysosome-associated membrane protein-1; Cq: Chloroquine; VEGFR2: Vascular endothelial growth factor receptor 2; KIF: Kinesin superfamily; SKIP: SifA and kinesin-interacting protein; MAPs: Microtubule-associated proteins; BORC: Multisubunit BLOC-1 complex; MTOC: Microtubule-organizing center; KBP: Kinesin binding protein; CTLs: Cytotoxic T lymphocytes; HER2: Epidermal growth factor receptor 2; M-6-P: Mannose6-phosphate; TGN: Trans-Golgi network; ILV: Intraluminal vesicles; MVB: Multivesicular bodies; TRPML: Transient receptor potential mucolipin; TPC: Two-pore channel; CLEAR: Coordinated lysosomal expression and regulation; RTK: Receptor tyrosine kinases; SNARE: Soluble $N$-ethylmaleimide-sensitive fusion attachment protein receptor; EGFR: Epidermal growth factor receptor; PDGFR: Platelet-derived growth factor receptor.
\end{abstract}

\section{Acknowledgments}

Not applicable.

\section{Authors' contributions}

SCY and XWB designed the study. TT and ZYY collected materials and wrote the first draft of the manuscript. TT and SCY modified the manuscript and drew illustrations. $X Y Y, L G$, and $Q W$ provided suggestions for modifying the article. JW helped draw the illustrations. DW assisted in translating the manuscript. LL, SCY, and TT revised the manuscript. All authors read and approved the final manuscript.

\section{Funding}

This study was supported by grants from the National Key Research and Development Program of China (2016YFA0202104 to SCY), the National Natural Science Foundation of China (81803029 to LL), the Key Natural Science Foundation of ChongQing (cstc2019jcyj-zdxmX0023 to LG), and the Key Clinical Research Program of Southwest Hospital (SWH2017ZDCX1003 to SCY).

Availability of data and materials

Not applicable.

Ethics approval and consent to participate

Not applicable.
Consent for publication

Not applicable.

\section{Competing interests}

The authors declare that they have no competing interests.

\section{Author details}

${ }^{1}$ Department of Stem Cell and Regenerative Medicine, Institute of Pathology and Southwest Cancer Center, Southwest Hospital, Third Military Medical University (Army Medical University), Chongqing 400038, China. ${ }^{2}$ Department of Hematology, Xinqiao Hospital, Third Military Medical University (Army Medical University), Chongqing 400037, China.

Received: 15 February 2020 Accepted: 29 October 2020

Published online: 18 November 2020

\section{References}

1. Ballabio A. The awesome lysosome. EMBO Mol Med. 2016;8(2):73-6.

2. Rink J, et al. Rab conversion as a mechanism of progression from early to late endosomes. Cell. 2005;122(5):735-49.

3. Hanson PI, Cashikar A. Multivesicular body morphogenesis. Annu Rev Cell Dev Biol. 2012:28:337-62.

4. Luzio JP, Pryor PR, Bright NA. Lysosomes: fusion and function. Nat Rev Mol Cell Biol. 2007;8(8):622-32.

5. Chakrabarti S, et al. Impaired membrane resealing and autoimmune myositis in synaptotagmin VII-deficient mice. J Cell Biol. 2003:162(4):543-9.

6. Medina DL, et al. Transcriptional activation of lysosomal exocytosis promotes cellular clearance. Dev Cell. 2011:21(3):421-30.

7. Galluzzi $\mathrm{L}$, et al. Molecular definitions of autophagy and related processes. EMBO J. 2017;36(13):1811-36.

8. Yu L, Chen Y, Tooze SA. Autophagy pathway: cellular and molecular mechanisms. Autophagy. 2018;14(2):207-15.

9. Takats $\mathrm{S}$, et al. Interaction of the HOPS complex with Syntaxin 17 mediates autophagosome clearance in Drosophila. Mol Biol Cell. 2014;25(8):1338-54

10. Wurmser AE, Sato TK, Emr SD. New component of the vacuolar class C-Vps complex couples nucleotide exchange on the Ypt7 GTPase to SNARE-dependent docking and fusion. J Cell Biol. 2000;151(3):551-62.

11. Itakura E, Kishi-Itakura C, Mizushima N. The hairpin-type tail-anchored SNARE syntaxin 17 targets to autophagosomes for fusion with endosomes/lysosomes. Cell. 2012;151(6):1256-69.

12. Li SC, Kane PM. The yeast lysosome-like vacuole: endpoint and crossroads. Biochim Biophys Acta. 2009:1793(4):650-63.

13. Polishchuk EV, et al. Wilson disease protein ATP7B utilizes lysosomal exocytosis to maintain copper homeostasis. Dev Cell. 2014;29(6):686-700.

14. Grimm C, et al. Endolysosomal cation channels and cancer-A link with great potential. Pharmaceuticals (Basel). 2018;11(1):4.

15. Lyu $L$, et al. TBBPA regulates calcium-mediated lysosomal exocytosis and thereby promotes invasion and migration in hepatocellular carcinoma. Ecotoxicol Environ Saf. 2020;192:110255.

16. Murley A, et al. Ltc1 is an ER-localized sterol transporter and a component of ER-mitochondria and ER-vacuole contacts. J Cell Biol. 2015;209(4):539-48.

17. Thelen AM, Zoncu R. Emerging roles for the lysosome in lipid metabolism. Trends Cell Biol. 2017;27(11):833-50.

18. Elbaz-Alon Y, et al. A dynamic interface between vacuoles and mitochondria in yeast. Dev Cell. 2014;30(1):95-102.

19. Honscher $C$, et al. Cellular metabolism regulates contact sites between vacuoles and mitochondria. Dev Cell. 2014;30(1):86-94.

20. Kumar N, et al. VPS13A and VPS13C are lipid transport proteins differentially localized at ER contact sites. J Cell Biol. 2018;217(10):3625-39.

21. Gonzalez Montoro A, et al. Vps39 interacts with Tom40 to establish one of two functionally distinct vacuole-mitochondria contact sites. Dev Cell. 2018;45(5):621-636 e7.

22. Yang $\mathrm{H}$, et al. Mechanisms of mTORC1 activation by RHEB and inhibition by PRAS40. Nature. 2017;552(7685):368-73. 
23. Lawrence RE, et al. A nutrient-induced affinity switch controls mTORC1 activation by its Rag GTPase-Ragulator lysosomal scaffold. Nat Cell Biol. 2018;20(9):1052-63.

24. Sancak Y, et al. The Rag GTPases bind raptor and mediate amino acid signaling to mTORC1. Science. 2008;320(5882):1496-501.

25. Bar-Peled $L$, et al. A tumor suppressor complex with GAP activity for the Rag GTPases that signal amino acid sufficiency to mTORC1. Science. 2013;340(6136):1100-6.

26. Wolfson $\mathrm{RL}$, et al. KICSTOR recruits GATOR1 to the lysosome and is necessary for nutrients to regulate $\mathrm{MTORC} 1$. Nature. 2017;543(7645):438-42.

27. Lawrence RE, Zoncu R. The lysosome as a cellular centre for signalling, metabolism and quality control. Nat Cell Biol. 2019;21(2):133-42.

28. Goh LK, Sorkin A. Endocytosis of receptor tyrosine kinases. Cold Spring Harb Perspect Biol. 2013;5(5):a017459.

29. Yamazaki T, et al. Role of Grb2 in EGF-stimulated EGFR internalization. J Cell Sci. 2002;115(Pt 9):1791-802.

30. Kowanetz K, et al. Suppressors of T-cell receptor signaling Sts-1 and Sts-2 bind to $\mathrm{Cbl}$ and inhibit endocytosis of receptor tyrosine kinases. J Biol Chem. 2004;279(31):32786-95.

31. Ballabio A, Gieselmann V. Lysosomal disorders: from storage to cellular damage. Biochim Biophys Acta. 2009;1793(4):684-96.

32. Nixon RA. The role of autophagy in neurodegenerative disease. Nat Med. 2013;19(8):983-97.

33. Sharma J, et al. Lysosomes and brain health. Annu Rev Neurosci. 2018:41:255-76.

34. Eskelinen EL. Roles of LAMP-1 and LAMP-2 in lysosome biogenesis and autophagy. Mol Aspects Med. 2006;27(5-6):495-502.

35. Levy JMM, Towers CG, Thorburn A. Targeting autophagy in cancer. Nat Rev Cancer. 2017;17(9):528-42.

36. Pu J, et al. Mechanisms and functions of lysosome positioning. J Cell Sci. 2016;129(23):4329-39.

37. Romao S, Gannage M, Munz C. Checking the garbage bin for problems in the house, or how autophagy assists in antigen presentation to the immune system. Semin Cancer Biol. 2013;23(5):391-6.

38. Mah LY, Ryan KM. Autophagy and cancer. Cold Spring Harb Perspect Biol. 2012;4(1):a008821.

39. van Kasteren SI, Overkleeft HS. Endo-lysosomal proteases in antigen presentation. Curr Opin Chem Biol. 2014;23:8-15.

40. Settembre C, et al. Signals from the lysosome: a control centre for cellular clearance and energy metabolism. Nat Rev Mol Cell Biol. 2013;14(5):283-96.

41. Steffan JJ, et al. Na+/H+ exchangers and RhoA regulate acidic extracellular $\mathrm{pH}$-induced lysosome trafficking in prostate cancer cells. Traffic. 2009;10(6):737-53.

42. Damaghi $\mathrm{M}$, et al. Chronic acidosis in the tumour microenvironment selects for overexpression of LAMP2 in the plasma membrane. Nat Commun. 2015;6:8752.

43. Settembre $C$, et al. TFEB links autophagy to lysosomal biogenesis. Science. 2011;332(6036):1429-33.

44. Settembre C, et al. A lysosome-to-nucleus signalling mechanism senses and regulates the lysosome via mTOR and TFEB. EMBO J. 2012:31(5):1095-108.

45. Kalluri R. The biology and function of exosomes in cancer. J Clin Invest. 2016:126(4):1208-15.

46. Desdin-Mico G, Mittelbrunn M. Role of exosomes in the protection of cellular homeostasis. Cell Adhes Migr. 2017;11(2):127-34.

47. Ohshima K, et al. Let-7 microRNA family is selectively secreted into the extracellular environment via exosomes in a metastatic gastric cancer cell line. PLoS ONE. 2010;5(10):e13247.

48. Kirkegaard T, Jaattela M. Lysosomal involvement in cell death and cancer. Biochim Biophys Acta. 2009;1793(4):746-54.

49. Cardoso CM, et al. Depletion of kinesin 5B affects lysosomal distribution and stability and induces peri-nuclear accumulation of autophagosomes in cancer cells. PLoS ONE. 2009;4(2):e4424.

50. Groth-Pedersen L, et al. Identification of cytoskeleton-associated proteins essential for lysosomal stability and survival of human cancer cells. PLOS ONE. 2012;7(10):e45381.

51. Davidson SM, Vander Heiden MG. Critical functions of the lysosome in cancer biology. Annu Rev Pharmacol Toxicol. 2017;57:481-507.
52. Commisso C, et al. Macropinocytosis of protein is an amino acid supply route in Ras-transformed cells. Nature. 2013;497(7451):633-7.

53. Haigler HT, McKanna JA, Cohen S. Rapid stimulation of pinocytosis in human carcinoma cells A-431 by epidermal growth factor. J Cell Biol. 1979:83(1):82-90.

54. Mosesson Y, Mills GB, Yarden Y. Derailed endocytosis: an emerging feature of cancer. Nat Rev Cancer. 2008;8(11):835-50.

55. Perera RM, Bardeesy N. Pancreatic cancer metabolism: breaking it down to build it back up. Cancer Discov. 2015;5(12):1247-61.

56. Guo JY, et al. Autophagy provides metabolic substrates to maintain energy charge and nucleotide pools in Ras-driven lung cancer cells. Genes Dev. 2016;30(15):1704-17.

57. Wong PM, et al. Regulation of autophagy by coordinated action of mTORC1 and protein phosphatase 2A. Nat Commun. 2015;6:8048.

58. Roczniak-Ferguson A, et al. The transcription factor TFEB links mTORC1 signaling to transcriptional control of lysosome homeostasis. Sci Signal. 2012;5(228):ra42.

59. Sardiello $M$, et al. A gene network regulating lysosomal biogenesis and function. Science. 2009;325(5939):473-7.

60. Perera RM, et al. Transcriptional control of autophagy-lysosome function drives pancreatic cancer metabolism. Nature. 2015;524(7565):361-5.

61. McDuff FK, Turner SD. Jailbreak: oncogene-induced senescence and its evasion. Cell Signal. 2011;23(1):6-13.

62. Collado $\mathrm{M}$, et al. Tumour biology: senescence in premalignant tumours. Nature. 2005;436(7051):642.

63. Braig $\mathrm{M}$, et al. Oncogene-induced senescence as an initial barrier in lymphoma development. Nature. 2005;436(7051):660-5.

64. Chen Z, et al. Crucial role of p53-dependent cellular senescence in suppression of Pten-deficient tumorigenesis. Nature. 2005:436(7051):725-30

65. Dankort D, et al. A new mouse model to explore the initiation, progression, and therapy of BRAFV600E-induced lung tumors. Genes Dev. 2007;21(4):379-84.

66. Kurz DJ, et al. Senescence-associated (beta)-galactosidase reflects an increase in lysosomal mass during replicative ageing of human endothelial cells. J Cell Sci. 2000;113(Pt 20):3613-22.

67. Lee BY, et al. Senescence-associated beta-galactosidase is lysosomal beta-galactosidase. Aging Cell. 2006;5(2):187-95

68. Aird KM, Zhang R. Nucleotide metabolism, oncogene-induced senescence and cancer. Cancer Lett. 2015;356(2 Pt A):204-10.

69. Gey C, Seeger K. Metabolic changes during cellular senescence investigated by proton NMR-spectroscopy. Mech Ageing Dev. 2013;134(3-4):130-8.

70. Perez-Mancera PA, Young AR, Narita M. Inside and out: the activities of senescence in cancer. Nat Rev Cancer. 2014;14(8):547-58.

71. Ros S, Schulze A. Linking glycogen and senescence in cancer cells. Cell Metab. 2012;16(6):687-8.

72. Ivanov A, et al. Lysosome-mediated processing of chromatin in senescence. J Cell Biol. 2013;202(1):129-43.

73. Allison AC. Lysosomes in cancer cells. J Clin Pathol Suppl (R Coll Pathol). 1974;7:43-50

74. Guo JY, et al. Autophagy suppresses progression of K-ras-induced lung tumors to oncocytomas and maintains lipid homeostasis. Genes Dev. 2013;27(13):1447-61.

75. Rao $\mathrm{S}$, et al. A dual role for autophagy in a murine model of lung cancer Nat Commun. 2014:5:3056.

76. Xie $X$, et al. Atg7 overcomes senescence and promotes growth of BrafV600E-driven melanoma. Cancer Discov. 2015;5(4):410-23.

77. Levy J, et al. Intestinal inhibition of Atg7 prevents tumour initiation through a microbiome-influenced immune response and suppresses tumour growth. Nat Cell Biol. 2015;17(8):1062-73.

78. Santanam U, et al. Atg7 cooperates with Pten loss to drive prostate cancer tumor growth. Genes Dev. 2016;30(4):399-407.

79. Yeo SK, et al. Autophagy differentially regulates distinct breast cancer stem-like cells in murine models via EGFR/Stat3 and Tgfbeta/Smad signaling. Cancer Res. 2016;76(11):3397-410.

80. Morelli MB, et al. Overexpression of transient receptor potential mucolipin-2 ion channels in gliomas: role in tumor growth and progression. Oncotarget. 2016;7(28):43654-68. 
81. Lamouille S, Xu J, Derynck R. Molecular mechanisms of epithelial-mesenchymal transition. Nat Rev Mol Cell Biol. 2014;15(3):178-96.

82. Liu H, et al. SPHK1 (sphingosine kinase 1) induces epithelial-mesenchymal transition by promoting the autophagy-linked lysosomal degradation of $\mathrm{CDH1} / \mathrm{E}-$ cadherin in hepatoma cells. Autophagy. 2017;13(5):900-13.

83. Zhang W, et al. LnCRNA CPS1-IT1 suppresses EMT and metastasis of colorectal cancer by inhibiting hypoxia-induced autophagy through inactivation of HIF-1alpha. Biochimie. 2018:144:21-7.

84. Wu WJ, Hirsch DS. Mechanism of E-cadherin lysosomal degradation. Nat Rev Cancer. 2009;9(2):143; author reply 143.

85. Fiore LS, et al. c-Abl and Arg induce cathepsin-mediated lysosomal degradation of the NM23-H1 metastasis suppressor in invasive cancer. Oncogene. 2014;33(36):4508-20.

86. Sharifi MN, et al. Autophagy promotes focal adhesion disassembly and cell motility of metastatic tumor cells through the direct interaction of paxillin with LC3. Cell Rep. 2016;15(8):1660-72.

87. Lock R, et al. Autophagy-dependent production of secreted factors facilitates oncogenic RAS-driven invasion. Cancer Discov. 2014;4(4):466-79.

88. Endres $\mathrm{M}$, et al. Regulation of matrix metalloproteinases (MMPs) expression and secretion in MDA-MB-231 breast cancer cells by LIM and SH3 protein 1 (LASP1). Oncotarget. 2016;7(39):64244-59.

89. Mohsen $A$, et al. A new gallium complex inhibits tumor cell invasion and matrix metalloproteinase MMP-14 expression and activity. Metallomics. 2017;9(8):1176-84.

90. Arvatz $\mathrm{G}$, et al. The heparanase system and tumor metastasis: is heparanase the seed and soil? Cancer Metastasis Rev. 2011;30(2):253-68.

91. Withana NP, et al. Cathepsin B inhibition limits bone metastasis in breast cancer. Cancer Res. 2012;72(5):1199-209.

92. Keliher EJ, et al. Targeting cathepsin $\mathrm{E}$ in pancreatic cancer by a small molecule allows in vivo detection. Neoplasia. 2013;15(7):684-93.

93. Small DM, et al. Cathepsin S from both tumor and tumor-associated cells promote cancer growth and neovascularization. Int J Cancer. 2013;133(9):2102-12.

94. Nguyen ON, et al. Two-pore channel function is crucial for the migration of invasive cancer cells. Cancer Res. 2017;77(6):1427-38.

95. Grimm C, et al. High susceptibility to fatty liver disease in two-pore channel 2-deficient mice. Nat Commun. 2014;5:4699.

96. Agarwal AK, et al. Role of tumor cell surface lysosome-associated membrane protein-1 (LAMP1) and its associated carbohydrates in lung metastasis. J Cancer Res Clin Oncol. 2015;141(9):1563-74.

97. Rusanen $\mathrm{M}$, et al. Heart diseases and long-term risk of dementia and Alzheimer's disease: a population-based CAIDE study. J Alzheimers Dis. 2014;42(1):183-91.

98. Kanao H, et al. Overexpression of LAMP3/TSC403/DC-LAMP promotes metastasis in uterine cervical cancer. Cancer Res. 2005;65(19):8640-5.

99. Wang D, et al. LAMP3 expression correlated with poor clinical outcome in human ovarian cancer. Tumour Biol. 2017;39(3):1010428317695014.

100. Xiao M, et al. LAPTM4B predicts axillary lymph node metastasis in breast cancer and promotes breast cancer cell aggressiveness in vitro. Cell Physiol Biochem. 2017;41(3):1072-82.

101. Furuta $\mathrm{K}$, et al. Expression of lysosome-associated membrane proteins in human colorectal neoplasms and inflammatory diseases. Am J Pathol. 2001;159(2):449-55.

102. Morgan MJ, et al. Metastatic cells are preferentially vulnerable to lysosomal inhibition. Proc Natl Acad Sci USA. 2018:115(36):E8479-88.

103. Folkman J, et al. Induction of angiogenesis during the transition from hyperplasia to neoplasia. Nature. 1989;339(6219):58-61.

104. Carmeliet P. Angiogenesis in health and disease. Nat Med. 2003;9(6):653-60.

105. Joyce JA, et al. Cathepsin cysteine proteases are effectors of invasive growth and angiogenesis during multistage tumorigenesis. Cancer Cell. 2004:5(5):443-53.

106. Kallunki T, Olsen OD, Jaattela M. Cancer-associated lysosomal changes: friends or foes? Oncogene. 2013;32(16):1995-2004.

107. Jiang $\mathrm{H}$, et al. Cathepsin K-mediated Notch1 activation contributes to neovascularization in response to hypoxia. Nat Commun. 2014;5:3838.

108. Jopling HM, et al. Endosome-to-plasma membrane recycling of VEGFR2 receptor tyrosine kinase regulates endothelial function and blood vessel formation. Cells. 2014;3(2):363-85.
109. Favia A, et al. VEGF-induced neoangiogenesis is mediated by NAADP and two-pore channel-2-dependent Ca2+ signaling. Proc Natl Acad Sci USA. 2014;111(44):E4706-15.

110. Intlekofer AM, Thompson CB. At the bench: preclinical rationale for CTLA-4 and PD-1 blockade as cancer immunotherapy. J Leukoc Biol. 2013;94(1):25-39.

111. Wang H, Han X, Xu J. Lysosome as the black hole for checkpoint molecules. Adv Exp Med Biol. 2020;1248:325-46.

112. Walker LS, Sansom DM. Confusing signals: recent progress in CTLA-4 biology. Trends Immunol. 2015;36(2):63-70.

113. Shiratori T, et al. Tyrosine phosphorylation controls internalization of CTLA-4 by regulating its interaction with clathrin-associated adaptor complex AP-2. Immunity. 1997;6(5):583-9.

114. Schneider $\mathrm{H}$, et al. Cytolytic T lymphocyte-associated antigen-4 and the TCR zeta/CD3 complex, but not CD28, interact with clathrin adaptor complexes AP-1 and AP-2. J Immunol. 1999;163(4):1868-79.

115. Bossi G, Griffiths GM. Degranulation plays an essential part in regulating cell surface expression of Fas ligand in T cells and natural killer cells. Nat Med. 1999;5(1):90-6.

116. Chuang E, et al. Interaction of CTLA-4 with the clathrin-associated protein AP50 results in ligand-independent endocytosis that limits cell surface expression. J Immunol. 1997;159(1):144-51.

117. lida T, et al. Regulation of cell surface expression of CTLA-4 by secretion of CTLA-4-containing lysosomes upon activation of CD4+ T cells. J Immunol. 2000;165(9):5062-8.

118. Pentcheva-Hoang T, et al. Programmed death-1 concentration at the immunological synapse is determined by ligand affinity and availability. Proc Natl Acad Sci USA. 2007;104(45):17765-70.

119. Casey TM, Meade JL, Hewitt EW. Organelle proteomics: identification of the exocytic machinery associated with the natural killer cell secretory lysosome. Mol Cell Proteomics. 2007;6(5):767-80.

120. Daniele T, et al. A role for Rab7 in the movement of secretory granules in cytotoxic T lymphocytes. Traffic. 2011;12(7):902-11.

121. Akalay I, et al. Epithelial-to-mesenchymal transition and autophagy induction in breast carcinoma promote escape from T-cell-mediated lysis. Cancer Res. 2013;73(8):2418-27.

122. Baginska J, et al. Granzyme B degradation by autophagy decreases tumor cell susceptibility to natural killer-mediated lysis under hypoxia. Proc Natl Acad Sci USA. 2013;110(43):17450-5.

123. Tofilon PJ, Fike JR. The radioresponse of the central nervous system: a dynamic process. Radiat Res. 2000;153(4):357-70.

124. Samie M, et al. A TRP channel in the lysosome regulates large particle phagocytosis via focal exocytosis. Dev Cell. 2013;26(5):511-24.

125. Aras S, Zaidi MR. TAMeless traitors: macrophages in cancer progression and metastasis. Br J Cancer. 2017;117(11):1583-91.

126. Sun $L$, et al. Novel role of TRPML 2 in the regulation of the innate immune response. J Immunol. 2015;195(10):4922-32.

127. Plesch $E$, et al. Selective agonist of TRPML2 reveals direct role in chemokine release from innate immune cells. Elife. 2018;7:e39720.

128. Goodridge JP, et al. Remodeling of secretory lysosomes during education tunes functional potential in NK cells. Nat Commun. 2019;10(1):514.

129. Jongsma ML, et al. An ER-associated pathway defines endosomal architecture for controlled cargo transport. Cell. 2016;166(1):152-66.

130. Matteoni R, Kreis TE. Translocation and clustering of endosomes and lysosomes depends on microtubules. J Cell Biol. 1987;105(3):1253-65.

131. Hollenbeck PJ, Swanson JA. Radial extension of macrophage tubular lysosomes supported by kinesin. Nature. 1990;346(6287):864-6.

132. Harada A, et al. Golgi vesiculation and lysosome dispersion in cells lacking cytoplasmic dynein. J Cell Biol. 1998;141(1):51-9.

133. Heuser J. Changes in lysosome shape and distribution correlated with changes in cytoplasmic pH. J Cell Biol. 1989;108(3):855-64.

134. Parton RG, et al. pH-induced microtubule-dependent redistribution of late endosomes in neuronal and epithelial cells. J Cell Biol. 1991;113(2):261-74.

135. Maylin C, et al. Possibilities of radiotherapy in the local-regional treatment of breast cancer in 1981. Soins Gynecol Obstet Pueric. 1981:4:15-8.

136. Baas PW, et al. Polarity orientation of microtubules in hippocampal neurons: uniformity in the axon and nonuniformity in the dendrite. Proc Natl Acad Sci USA. 1988;85(21):8335-9. 
137. Yau KW, et al. Dendrites in vitro and in vivo contain microtubules of opposite polarity and axon formation correlates with uniform plus-endout microtubule orientation. J Neurosci. 2016:36(4):1071-85.

138. Rosa-Ferreira C, Munro S. Arl8 and SKIP act together to link lysosomes to kinesin-1. Dev Cell. 2011:21(6):1171-8.

139. Tanaka $Y$, et al. Targeted disruption of mouse conventional kinesin heavy chain, kif5B, results in abnormal perinuclear clustering of mitochondria. Cell. 1998;93(7):1147-58.

140. Nakata T, Hirokawa N. Point mutation of adenosine triphosphate-binding motif generated rigor kinesin that selectively blocks anterograde lysosome membrane transport. J Cell Biol. 1995;131(4):1039-53.

141. Loubery S, et al. Different microtubule motors move early and late endocytic compartments. Traffic. 2008:9(4):492-509.

142. Brown $\mathrm{CL}$, et al. Kinesin-2 is a motor for late endosomes and lysosomes. Traffic. 2005;6(12):1114-24.

143. Korolchuk VI, et al. Lysosomal positioning coordinates cellular nutrient responses. Nat Cell Biol. 2011;13(4):453-60.

144. Bentley $M$, et al. A novel assay reveals preferential binding between Rabs, kinesins, and specific endosomal subpopulations. J Cell Biol. 2015;208(3):273-81.

145. Matsushita M, et al. A novel kinesin-like protein, KIF1Bbeta3 is involved in the movement of lysosomes to the cell periphery in non-neuronal cells. Traffic. 2004;5(3):140-51.

146. Santama N, et al. KIF2beta, a new kinesin superfamily protein in nonneuronal cells, is associated with lysosomes and may be implicated in their centrifugal translocation. EMBO J. 1998;17(20):5855-67.

147. Boucrot $E$, et al. The intracellular fate of Salmonella depends on the recruitment of kinesin. Science. 2005;308(5725):1174-8.

148. Hofmann I, Munro S. An N-terminally acetylated Arf-like GTPase is localised to lysosomes and affects their motility. J Cell Sci. 2006;119(Pt 8):1494-503.

149. Pu J, et al. BORC, a multisubunit complex that regulates lysosome positioning. Dev Cell. 2015;33(2):176-88.

150. Raiborg C, et al. ER-endosome contact sites in endosome positioning and protrusion outgrowth. Biochem Soc Trans. 2016;44(2):441-6.

151. Dehmelt L, Halpain S. The MAP2/Tau family of microtubule-associated proteins. Genome Biol. 2005;6(1):204

152. Al-Bassam J, et al. Analysis of the weak interactions of ADP-Unc104 and ADP-kinesin with microtubules and their inhibition by MAP2c. Cell Motil Cytoskelet. 2007;64(5):377-89.

153. Lipka J, et al. Microtubule-binding protein doublecortin-like kinase 1 (DCLK1) guides kinesin-3-mediated cargo transport to dendrites. EMBO J. 2016;35(3):302-18.

154. Sung $H$ H, et al. Drosophila ensconsin promotes productive recruitment of Kinesin-1 to microtubules. Dev Cell. 2008;15(6):866-76.

155. Kevenaar JT, et al. Kinesin-binding protein controls microtubule dynamics and cargo trafficking by regulating Kinesin Motor Activity. Curr Biol. 2016;26(7):849-61.

156. Paschal BM, Vallee RB. Retrograde transport by the microtubule-associated protein MAP 1C. Nature. 1987;330(6144):181-3.

157. Ishikawa T. Structural biology of cytoplasmic and axonemal dyneins. J Struct Biol. 2012;179(2):229-34.

158. Johansson $\mathrm{M}$, et al. Activation of endosomal dynein motors by stepwise assembly of Rab7-RILP-p150Glued, ORP1L, and the receptor betalll spectrin. J Cell Biol. 2007;176(4):459-71.

159. Rocha N, et al. Cholesterol sensor ORP1L contacts the ER protein VAP to control Rab7-RILP-p150 Glued and late endosome positioning. J Cell Biol. 2009;185(7):1209-25.

160. Li X, et al. A molecular mechanism to regulate lysosome motility for lysosome positioning and tubulation. Nat Cell Biol. 2016;18(4):404-17.

161. Huynh KK, et al. LAMP proteins are required for fusion of lysosomes with phagosomes. EMBO J. 2007:26(2):313-24

162. Krzewski K, et al. LAMP1/CD107a is required for efficient perforin delivery to lytic granules and NK-cell cytotoxicity. Blood. 2013;121(23):4672-83.
163. Schwenk BM et al. The FTLD risk factor TMEM106B and MAP6 control dendritic trafficking of lysosomes. EMBO J. 2014;33(5):450-67.

164. Encalada SE, et al. Stable kinesin and dynein assemblies drive the axonal transport of mammalian prion protein vesicles. Cell. 2011:144(4):551-65.

165. Kamiyama R, Saikawa M, Kishimoto S. Significance of retropharyngeal lymph node dissection in hypopharyngeal cancer. Jpn J Clin Oncol. 2009;39(10):632-7.

166. D'Costa VM, et al. Salmonella disrupts host endocytic trafficking by SopD2-mediated inhibition of Rab7. Cell Rep. 2015;12(9):1508-18.

167. Saric A, et al. mTOR controls lysosome tubulation and antigen presentation in macrophages and dendritic cells. Mol Biol Cell. 2016;27(2):321-33.

168. Vyas JM, et al. Tubulation of class II MHC compartments is microtubule dependent and involves multiple endolysosomal membrane proteins in primary dendritic cells. J Immunol. 2007;178(11):7199-210.

169. Dell'Angelica EC, et al. Lysosome-related organelles. FASEB J. 2000;14(10):1265-78

170. Blott EJ, Griffiths GM. Secretory lysosomes. Nat Rev Mol Cell Biol. 2002:3(2):122-31

171. Stinchcombe JC, et al. Centrosome polarization delivers secretory granules to the immunological synapse. Nature. 2006:443(7110):462-5.

172. Neeft M, et al. Munc13-4 is an effector of rab27a and controls secretion of lysosomes in hematopoietic cells. Mol Biol Cell. 2005;16(2):731-41.

173. Kroemer $G$, Jaattela M. Lysosomes and autophagy in cell death control. Nat Rev Cancer. 2005;5(11):886-97.

174. Nishimura Y, et al. Overexpression of ROCK in human breast cancer cells: evidence that ROCK activity mediates intracellular membrane traffic of lysosomes. Pathol Oncol Res. 2003;9(2):83-95.

175. Nishimura Y, et al. A role for small GTPase RhoA in regulating intracellular membrane traffic of lysosomes in invasive rat hepatoma cells. Histochem J. 2002;34(5):189-213.

176. Glunde K, et al. Extracellular acidification alters lysosomal trafficking in human breast cancer cells. Neoplasia. 2003;5(6):533-45.

177. Steffan JJ, et al. Supporting a role for the GTPase Rab7 in prostate cancer progression. PLoS ONE. 2014;9(2):e87882.

178. Reddy A, Caler EV, Andrews NW. Plasma membrane repair is mediated by $\mathrm{Ca}(2+)$-regulated exocytosis of lysosomes. Cell. 2001;106(2):157-69.

179. Rodriguez A, et al. Lysosomes behave as Ca2+-regulated exocytic vesicles in fibroblasts and epithelial cells. J Cell Biol. 1997;137(1):93-104

180. Andrews NW, Almeida PE, Corrotte M. Damage control: cellular mechanisms of plasma membrane repair. Trends Cell Biol. 2014:24(12):734-42.

181. Mohamed MM, Sloane BF. Cysteine cathepsins: multifunctional enzymes in cancer. Nat Rev Cancer. 2006;6(10):764-75.

182. Dykes SS, et al. The Arf-like GTPase Arl8b is essential for three-dimensional invasive growth of prostate cancer in vitro and xenograft formation and growth in vivo. Oncotarget. 2016:7(21):31037-52.

183. Macpherson IR, et al. CLIC3 controls recycling of late endosomal MT1MMP and dictates invasion and metastasis in breast cancer. J Cell Sci. 2014;127(Pt 18):3893-901

184. Monteiro $\mathrm{P}$, et al. Endosomal WASH and exocyst complexes control exocytosis of MT1-MMP at invadopodia. J Cell Biol. 2013;203(6):1063-79.

185. Dozynkiewicz MA, et al. Rab25 and CLIC3 collaborate to promote integrin recycling from late endosomes/lysosomes and drive cancer progression. Dev Cell. 2012;22(1):131-45.

186. Circu ML, et al. Correction: a novel high content imaging-based screen identifies the anti-helminthic niclosamide as an inhibitor of lysosome anterograde trafficking and prostate cancer cell invasion. PLOS ONE. 2016;11(3):e0151718.

\section{Publisher's Note}

Springer Nature remains neutral with regard to jurisdictional claims in published maps and institutional affiliations. 